\title{
Erratum: Quantum metrology with nonequilibrium steady states of quantum spin chains [Phys. Rev. A 90, 062130 (2014)]
}

\author{
Ugo Marzolino and Tomaž Prosen \\ (Received 25 August 2016; published 27 September 2016)
}

DOI: 10.1103/PhysRevA.94.039903

We realized there was a combinatorial mistake in our paper. Before explaining and correcting the mistake, we stress that it does not affect the qualitative interpretation of the results.

The mistake is in Eq. (31) of our previous paper, namely, the leading order in the dissipation strength of the Fisher information with respect to the anisotropy for rational $\frac{\eta}{\pi}=\frac{\arccos \Delta}{\pi}=\frac{q}{p}$ and coprime integers $p, q$ provided $|\Delta|<1$. The correct formulation is

$$
\begin{aligned}
\sum_{k=1}^{n}\left\langle L\left|T^{k-1} D T^{n-k}\right| R\right\rangle= & \sum_{k=1}^{n} \sum_{j=1}^{d} \sum_{l=1}^{d+1}\left\langle L\left|\left(T^{(d)}\right)^{k-1}\right| j\right\rangle\langle j|D| l\rangle\left\langle l\left|\left(T^{(d)}\right)^{n-k}\right| R\right\rangle \\
= & \sum_{k=1}^{n} \sum_{j, l=1}^{d}\left\langle L\left|V^{(d)}\left(T_{J}^{(d)}\right)^{k-1}\left(V^{(d)}\right)^{-1}\right| j\right\rangle\langle j|D| l\rangle\left\langle l\left|V^{(d)}\left(T_{J}^{(d)}\right)^{n-k}\left(V^{(d)}\right)^{-1}\right| R\right\rangle \\
& +\sum_{k=1}^{n} \sum_{l=1}^{n-k-d}\left\langle L\left|V^{(d)}\left(T_{J}^{(d)}\right)^{k-1}\left(V^{(d)}\right)^{-1}\right| d\right\rangle\langle d|D| d+1\rangle\left\langle d+1\left|T^{l-1}\right| d+1\right\rangle \\
& \times\langle d+1|T| d\rangle\left\langle d\left|V^{(d)}\left(T_{J}^{(d)}\right)^{n-k-l}\left(V^{(d)}\right)^{-1}\right| R\right\rangle \\
= & \frac{d}{2\left(1-\Delta^{2}\right)(d+1)}\left(n \sum_{j, l=1}^{d}\langle j|D| l\rangle\left\langle L\left|V^{-1}\right| j\right\rangle \psi_{l}+\frac{1}{16}\left[n^{2}-n(2 d+1)\right]\right. \\
& \left.\times(d+1)^{2} \sin ^{2}(d \eta)\left\langle L\left|V^{-1}\right| d\right\rangle \psi_{d}\right)+O(1) \\
\equiv & \xi_{2} n^{2}+\xi_{1} n+O(1),
\end{aligned}
$$

where $d=|p|-1$. The difference with respect to Eq. (31) in our previous paper is in the third line. There is an additional sum $\sum_{l=1}^{n-k-d}$ together with the contribution $T^{l-1}$. Finally the leading order of the Fisher information for small $\frac{\lambda}{J}$ and large $n$ is

$$
\begin{aligned}
F_{\Delta}^{(0)} & \left.=\frac{\lambda^{2} \mu^{2}}{J^{2}}[\tilde{\xi}] n^{2}+\xi n+O(1)\right], \text { with } \\
\xi & =\frac{1}{2\left(1-\Delta^{2}\right)}\left(\xi_{1}+\frac{1}{4} \frac{d^{2} \chi}{d \eta^{2}}\right), \\
\widetilde{\xi} & =\frac{\xi_{2}}{2\left(1-\Delta^{2}\right)}
\end{aligned}
$$

The new quadratic term $\widetilde{\xi} n^{2}$ does not change the interpretation of the results. Indeed, the quantum Fisher information detects both enhanced metrological performances and critical behaviors when it is superextensive. Due to the perturbative analysis, valid for $\frac{\lambda \mu}{J}<\frac{1}{\sqrt{n}}$ and $|\Delta|<1$, see Eq. (7) of the original paper, the Fisher information cannot be superextensive for rational $\frac{\eta}{\pi}$ in agreement with the statement in the original paper. Moreover, the coefficient $\tilde{\xi}$ of the quadratic term is a regular function of the anisotropy $\Delta$ as shown in Fig. 1. On the other hand, the corrected coefficient $\xi$ of the linear term exhibits the same fractal-like structure described in the original paper, depicted in Fig. 2: Closer zooms reveal additional cusplike patterns. The term that explodes for large $|p|$ and that dominates for irrational $\frac{\eta}{\pi}$ is indeed the double sum in the fourth line of (31), which contributes to $\xi$ and which is not amended here. Finally, both coefficients $\xi$ and $\tilde{\xi}$ diverge approaching the isotropic point $|\Delta|=1$.

Finally, the variable $\epsilon$ in the equation in the text soon after Eq. (9) of our original paper should be substituted with $\lambda / 2$. 


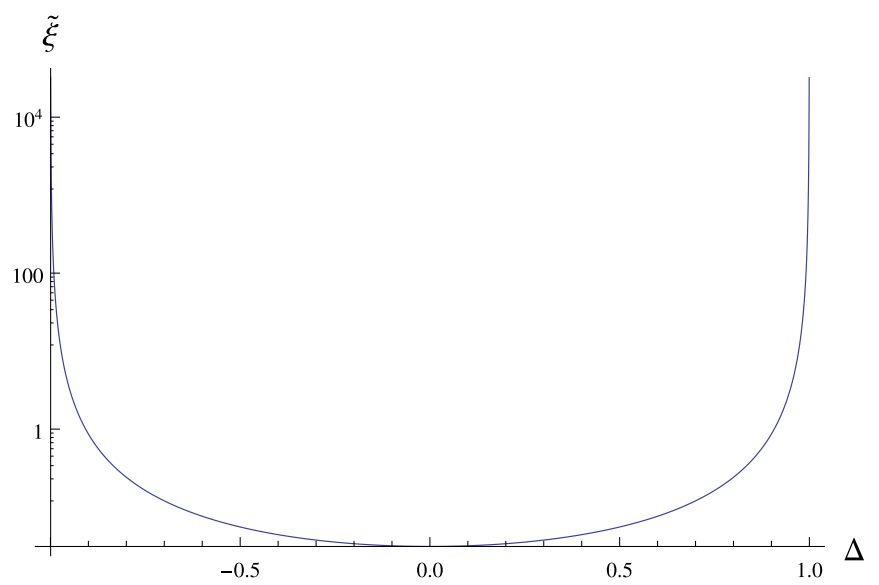

FIG. 1. Semilogarithmic plot of the quadratic (in $n$ ) coefficient $\widetilde{\xi}$ of the Fisher information as a function of the anisotropy $\Delta \in[-1,1]$ for rational $\frac{\eta}{\pi}$.

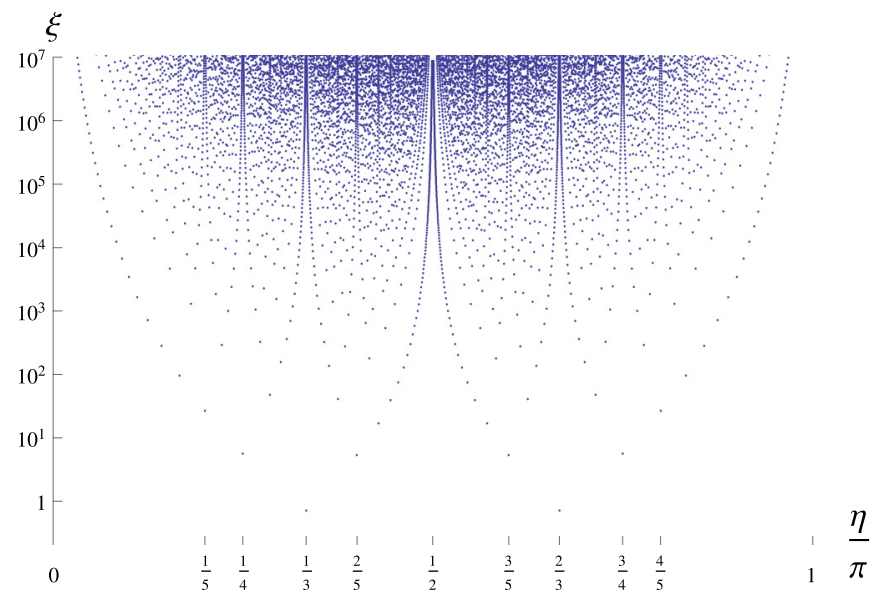

FIG. 2. Semilogarithmic plot of the linear (in $n$ ) coefficient $\xi$ of the Fisher information as a function of the anisotropy parameter $\frac{\eta}{\pi} \in[0,1]$ for rational $\frac{\eta}{\pi}$.

The work has been supported by Grants No. P1-0044 and No. J1-5439 of the Slovenian Research Agency. 